\title{
Research Article Symmetries, Conservation Laws, and Wave Equation on the Milne Metric
}

\author{
Ahmad M. Ahmad, Ashfaque H. Bokhari, and F. D. Zaman \\ Department of Mathematics and Statistics, King Fahd University of Petroleum and Minerals, \\ Dhahran 31261, Saudi Arabia \\ Correspondence should be addressed to Ashfaque H. Bokhari, abokhari@kfupm.edu.sa
}

Received 2 September 2012; Revised 27 November 2012; Accepted 27 November 2012

Academic Editor: Fazal M. Mahomed

Copyright (C) 2012 Ahmad M. Ahmad et al. This is an open access article distributed under the Creative Commons Attribution License, which permits unrestricted use, distribution, and reproduction in any medium, provided the original work is properly cited.

\begin{abstract}
Noether symmetries provide conservation laws that are admitted by Lagrangians representing physical systems. For partial differential equation possessing Lagrangians these symmetries are obtained by the invariance of the corresponding action integral. In this paper we provide a systematic procedure for determining Noether symmetries and conserved vectors for a Lagrangian constructed from a Lorentzian metric of interest in mathematical physics. For completeness, we give Lie point symmetries and conservation laws admitted by the wave equation on this Lorentzian metric.
\end{abstract}

\section{Introduction}

A vast amount of work is available on analyzing differential equations (DEs) through their Lie point symmetries. These symmetries are important in that they play pivotal role in solving nonlinear differential equations. Apart from Lie point symmetries, there are other interesting symmetries that are associated with differential equations which possess Lagrangians. These symmetries are called Noether symmetries and describe physical features of DEs in terms of conservation laws they admit. The connection between symmetry and conservation laws has been inherent in mathematical physics since Emmy Noether published her classical work linking the two [1]. Noether proved that for every infinitesimal transformation admitted by the action integral of a Lagrangian system, there exists a conservation law [1]. The relationship between symmetries and conservation laws in the absence of a Lagrangian is detailed in [2,3] and references therein. Extending some of the earlier work, Bokhari et al. $[4,5]$ investigated Noether symmetries for the actions of certain line elements associated with the Lagrangian of some Lorentzian metrics of signature 2. More recently, extending 
the work in $[4,5]$, new results are obtained for existence of conserved quantities for spaces of different curvatures [6]. In the present study, we revisit the work in [6] and investigate Noether symmetries of the Euler Lagrange equations of a Lorentzian metric, known as the Milne Model [7] whose metric is given by

$$
d s^{2}=-d t^{2}+t^{2}\left(d x^{2}+e^{2 x}\left(d y^{2}+d z^{2}\right)\right)
$$

The Milne metric represents an empty universe and is of interest in special relativity. Our reason to choose this metric for the present study is that whereas it is zero curvature, it illustrates some features that have been associated with the expanding universe implicit in special relativity. The plan of the paper is as follows.

In the next section we find Noether symmetries of the Lagrangian constructed from (1.1). In Section 2, we construct the wave equation for the Milne metric (1.1) and then find its Noether symmetries. In the third section, the Lie symmetries of the wave on Milne metric are compared with those of the Noether symmetries obtained from its lagrangian. For details of Noether and Lie symmetries, we refer the reader to, inter alia, [8-11].

\section{Invariance of the Geodesic Equations and Noether Symmetries}

Geodesic equations are the Euler Lagrange equations determined from invariance of an action integral [4]. In order to find Noether symmetries admitted by the geodesic equations for the Milne metric, we write a Lagrangian, $L=L(s, t, x, y, z, \dot{t}, \dot{x}, \dot{y}, \dot{z})$, that can be constructed by the Milne metric and given by the expression

$$
L=-\dot{t}^{2}+t^{2}\left(\dot{x}^{2}+e^{2 x}\left(\dot{y}^{2}+\dot{z}^{2}\right)\right)
$$

The general Noether symmetry generators corresponding to this Lagrangian are [1]

$$
X=\xi \frac{\partial}{\partial s}+\eta^{1} \frac{\partial}{\partial t}+\eta^{2} \frac{\partial}{\partial x}+\eta^{3} \frac{\partial}{\partial y}+\eta^{4} \frac{\partial}{\partial z}+\dot{\eta}^{1} \frac{\partial}{\partial \dot{t}}+\dot{\eta}^{2} \frac{\partial}{\partial \dot{x}}+\dot{\eta}^{3} \frac{\partial}{\partial \dot{y}}+\dot{\eta}^{4} \frac{\partial}{\partial \dot{z}^{\prime}}
$$

where $\xi, \eta^{1}, \eta^{2}, \eta^{3}, \eta^{4}$ are functions of $s, t, x, y, z$, and are given by

$$
X L+L D_{s} \xi=D_{s} f
$$


where $f(x, y, z, t)$ is a gauge function. The resultant over determined system of partial differential equations, after separation by monomials, is

$$
\begin{aligned}
& \xi_{t}=0, \quad \xi_{x}=0, \quad \xi_{y}=0, \\
& \xi_{z}=0, \quad 2 \eta_{t}^{1}-\xi_{s}=0, \quad 2 \eta^{1}+2 t \eta_{x}^{2}-t \xi_{s}=0 \\
& 2 \eta^{1}+2 t \eta^{2}+2 t \eta_{y}^{3}-t \xi_{s}=0, \quad 2 \eta^{1}+2 t \eta^{2}+2 t \eta_{z}^{4}-t \xi_{s}=0 \\
& \eta_{x}^{1}-t^{2} \eta_{t}^{2}=0, \quad \eta_{y}^{1}-t^{2} e^{2 x} \eta_{t}^{3}=0, \quad \eta_{z}^{1}-t^{2} e^{2 x} \eta_{t}^{4}=0, \\
& \eta_{y}^{2}+e^{2 x} \eta_{x}^{3}=0, \quad \eta_{z}^{2}+e^{2 x} \eta_{x}^{4}=0, \quad \eta_{z}^{3}+\eta_{y}^{4}=0 \\
& 2 \eta_{s}^{1}+f_{t}=0, \quad 2 t^{2} \eta_{s}^{2}-f_{x}=0, \quad 2 t^{2} e^{2 x} \eta_{s}^{3}-f_{y}=0 \\
& 2 t^{2} e^{2 x} \eta_{s}^{4}-f_{z}=0, \quad f_{s}=0 .
\end{aligned}
$$

Solving above system of equations iteratively, we obtain

$$
\begin{aligned}
\xi= & \frac{1}{2} C_{1} s^{2}+C_{2} s+C_{3} \\
\eta^{1}= & \frac{1}{2} t s C_{1}+\frac{1}{2} t C_{2}-\frac{1}{2} s\left(e^{x}\left(y^{2}+z^{2}\right)+e^{-x}\right) C_{5}-\frac{1}{2} s z e^{x} C_{6} \\
& -\frac{1}{2} s e^{x} C_{7}-\frac{1}{2} s y e^{x} C_{8}+\left(e^{-x}+\left(y^{2}+z^{2}\right) e^{x}\right) C_{9}+z e^{x} C_{10} \\
& +e^{x} C_{11}+y e^{x} C_{12} \\
\eta^{2}= & \frac{1}{2 t}\left(\left(y^{2}+z^{2}\right) s e^{x}-s e^{-x}\right) C_{5}+\frac{1}{2 t} s z e^{x} C_{6}+\frac{1}{2 t} s e^{x} C_{7}+\frac{1}{2 t} s y e^{x} C_{8} \\
& +\frac{1}{t}\left(e^{-x}-\left(y^{2}+z^{2}\right) e^{x}\right) C_{9}-\frac{1}{t} z e^{x} C_{10}-\frac{1}{t} e^{x} C_{11}-\frac{1}{t} y e^{x} C_{12} \\
& +2 y C_{13}-z C_{14}-C_{15}, \\
\eta^{3}= & \frac{1}{t} s y e^{-x} C_{5}+\frac{1}{2 t} s e^{-x} C_{8}-\frac{2}{t} y e^{-x} C_{9}-\frac{1}{t} e^{-x} C_{12}+\left(e^{-2 x}-y^{2}+z^{2}\right) C_{13} \\
& +y z C_{14}+y C_{15}+z C_{16}+C_{17}, \\
\eta^{4}= & \frac{1}{t} s z e^{-x} C_{5}+\frac{1}{2} \frac{1}{t} s e^{-x} C_{6}-\frac{2}{t} z e^{-x} C_{9}-\frac{1}{t} e^{-x} C_{10}-2 y z C_{13} \\
& -\frac{1}{2}\left(e^{-2 x}+y^{2}-z^{2}\right) C_{14}+z C_{15}-y C_{16}+C_{18},
\end{aligned}
$$

where $C_{i}$ are arbitrary constants. The corresponding gauge term is given by

$$
f=-\frac{1}{2} t^{2} C_{1}+C_{4}+t\left(e^{-x}+\left(y^{2}+z^{2}\right) e^{x}\right) C_{5}+t z e^{x} C_{6}+t e^{x} C_{7}+t y e^{x} C_{8}
$$


Thus, a basis for the Lie algebra of Noether point symmetry generators is (only the associated nonzero gauge terms, $f$, are given)

$$
\begin{aligned}
X_{1}= & \frac{1}{2} s^{2} \frac{\partial}{\partial s}+\frac{1}{2} s t \frac{\partial}{\partial t^{\prime}}, \quad f=-\frac{1}{2} t^{2}, \\
X_{2}= & s \frac{\partial}{\partial s}+\frac{1}{2} t \frac{\partial}{\partial t^{\prime}}, \quad X_{3}=\frac{\partial}{\partial s^{\prime}} \\
X_{4}= & -\frac{1}{2} s\left(e^{x}\left(y^{2}+z^{2}\right)+e^{-x}\right) \frac{\partial}{\partial t}+\frac{1}{2 t}\left(\left(y^{2}+z^{2}\right) s e^{x}-s e^{-x}\right) \frac{\partial}{\partial x} \\
& +\frac{1}{t} s y e^{-x} \frac{\partial}{\partial y}+\frac{1}{t} s z e^{-x} \frac{\partial}{\partial z}, \quad f=t\left(e^{-x}+\left(y^{2}+z^{2}\right) e^{x}\right), \\
X_{5}= & -\frac{1}{2} s z e^{x} \frac{\partial}{\partial t}+\frac{1}{2 t} s z e^{x} \frac{\partial}{\partial x}+\frac{1}{2 t} s e^{-x} \frac{\partial}{\partial z}, \quad f=t z e^{x}, \\
X_{6}= & -\frac{1}{2} s e^{x} \frac{\partial}{\partial t}+\frac{1}{2 t} s e^{x} \frac{\partial}{\partial x}, \quad f=t e^{x}, \\
X_{7}= & -\frac{1}{2} s y e^{x} \frac{\partial}{\partial t}+\frac{1}{2 t} s y e^{x} \frac{\partial}{\partial x}+\frac{1}{2 t} s e^{-x} \frac{\partial}{\partial y}, \quad f=t y e^{x}, \\
X_{8}= & \left(e^{-x}+\left(y^{2}+z^{2}\right) e^{x}\right) \frac{\partial}{\partial t}+\frac{1}{t}\left(e^{-x}-\left(y^{2}+z^{2}\right) e^{x}\right) \frac{\partial}{\partial x}-\frac{2}{t} y e^{-x} \frac{\partial}{\partial y}-\frac{2}{t} z e^{-x} \frac{\partial}{\partial z}, \\
X_{13}= & -z \frac{\partial}{\partial x}+y z \frac{\partial}{\partial y}-\frac{1}{2}\left(e^{-2 x}+y^{2}-z^{2}\right) \frac{\partial}{\partial z}, \\
X_{14}= & z e^{x} \frac{\partial}{\partial t}-\frac{1}{t} z e^{x} \frac{\partial}{\partial x}-\frac{1}{t} e^{-x} \frac{\partial}{\partial z}, \quad X_{10}=e^{x} \frac{\partial}{\partial t}-\frac{1}{t} e^{x} \frac{\partial}{\partial x}, \\
X_{12}= & 2 y \frac{\partial}{\partial x}+\left(e^{-2 x}-y^{2}+z^{2}\right) \frac{\partial}{\partial y}-2 y z \frac{\partial}{\partial z}, \\
X_{11} & =\frac{1}{\partial t} y e^{x} \frac{\partial}{\partial x}-\frac{1}{t} e^{-x} \frac{\partial}{\partial y},
\end{aligned}
$$

The above symmetry generators form a closed Lie algebra of the symmetry group $G_{17}$. Further, each of these generators gives rise to a conservation law (first integral) of the geodesic equations via Noether's theorem. For example, the symmetry generators $X_{3}, X_{16}$, $X_{17}$ correspond to linear momentum conservation along $s, y$, and $z$ directions, respectively, 
while $X_{15}$ represents a hyperbolic rotation. Moreover, the conserved vector associated with $X_{3}[12]$ is

$$
\begin{aligned}
T & =\left(\xi \dot{t}-\eta^{1}\right) \frac{\partial L}{\partial \dot{t}}+\left(\xi \dot{x}-\eta^{2}\right) \frac{\partial L}{\partial \dot{x}}+\left(\xi \dot{y}-\eta^{3}\right) \frac{\partial L}{\partial \dot{y}}+\left(\xi \dot{z}-\eta^{4}\right) \frac{\partial L}{\partial \dot{z}}-\xi L+f(s, t, x, y, z) \\
& =s L+t \dot{t}
\end{aligned}
$$

Note that the conservation laws obtained above are 7 more than those given by the Killing vectors [13] of the Milne metric (the additional seven generators are $X_{1}$ to $X_{7}$ ).

\section{Wave Equation}

In this context it may be interesting to study the symmetries/conservation laws of the wave equation on the Milne metric. The wave equation on this metric can be given by using the box operator [13]:

$$
\square u=\frac{1}{\sqrt{|-g|}} \frac{\partial}{\partial x^{i}}\left(\sqrt{|-g|} g^{i j} \frac{\partial}{\partial x^{i}} u\right)=0
$$

Simplifying the above expression, wave equation takes the form

$$
u_{t t}-\frac{1}{t^{2}} u_{x x}-\frac{1}{t^{2} e^{2 x}} u_{y y}-\frac{1}{t^{2} e^{2 x}} u_{z z}=-\frac{3}{t} u_{t}+\frac{2}{t^{2}} u_{x}
$$

The Lie point symmetries of the wave equation (3.2) are given by the formula [9]

$$
X\left(\text { Equation (3.2) }\left.\right|_{\text {Equation (3.2) }}\right)=0,
$$

where $X$ is the prolonged symmetry generator given by

$$
X=\eta \partial_{u}+\xi^{4} \partial_{t}+\xi^{1} \partial_{x}+\xi^{2} \partial_{y}+\xi^{3} \partial_{z}
$$


Following the method adopted in Section 2, (3.4) gives an overdetermined system of determining equations in four unknowns. This system yields a 16-dimensional Lie symmetry group $G_{16}$ whose basis is given by

$$
\begin{aligned}
& X_{1}=t \partial_{t}, \\
& X_{2}=-2 t u e^{x} \partial_{u}+\left(1+t^{2}\right) e^{x} \partial_{t}+\frac{1}{t}\left(-1+t^{2}\right) e^{x} \partial_{x} \\
& X_{3}=-2 t u e^{x} \partial_{u}+\left(-1+t^{2}\right) e^{x} \partial_{t}+\frac{1}{t}\left(1+t^{2}\right) e^{x} \partial_{x}, \quad X_{4}=\partial_{y}, \\
& X_{5}=\frac{1}{t} e^{-x} \partial_{y}-y e^{x} \partial_{t}+\frac{1}{t} y e^{x} \partial_{x} \\
& X_{6}=-2 t y u e^{x} \partial_{u}+t^{2} y e^{x} \partial_{t}+t e^{-x} \partial_{y}+t y e^{x} \partial_{x} \\
& X_{7}=\partial_{z}, \quad X_{8}=\frac{1}{t} e^{-x} \partial_{z}-z e^{x} \partial_{t}+\frac{1}{t} z e^{x} \partial_{x} \\
& X_{9}=-2 t z u e^{x} \partial_{u}+t^{2} z e^{x} \partial_{t}+t e^{-x} \partial_{z}+t z e^{x} \partial_{x}, \quad X_{10}=y \partial_{z}-z \partial_{y}, \\
& X_{11}=-\partial_{x}+y \partial_{y}+z \partial_{z}, \quad X_{12}=-2 \partial_{x}+2 y \partial_{y}+2 z \partial_{z}+u \partial_{u} \\
& X_{13}=\frac{2}{t} y e^{-x} \partial_{y}+\frac{2}{t} z e^{-x} \partial_{z}+\left(-1-e^{2 x}\left(y^{2}+z^{2}\right)\right) e^{-x} \partial_{t}+\frac{1}{t}\left(-1+e^{2 x}\left(y^{2}+z^{2}\right)\right) e^{-x} \partial_{x} \\
& X_{14}=-2 t u\left(1+e^{2 x}\left(y^{2}+z^{2}\right)\right) e^{-x} \partial_{u}+2 t y e^{-x} \partial_{y}+2 t z e^{-x} \partial_{z}+t\left(-1+e^{2 x}\left(y^{2}+z^{2}\right)\right) e^{-x} \partial_{x} \\
& +t^{2}\left(1+e^{2 x}\left(y^{2}+z^{2}\right)\right) e^{-x} \partial_{t} \\
& X_{15}=2 y \partial_{x}-2 y z \partial_{z}+\left(e^{-2 x}-y^{2}+z^{2}\right) \partial_{y}, \quad X_{16}=-2 y z \partial_{y}+2 z \partial_{x}+\left(e^{-2 x}+y^{2}-z^{2}\right) \partial_{z}
\end{aligned}
$$

It may be worth mentioning that $X_{1}$ gives a scaling conservation law along $t$ direction, $X_{4}, X_{7}$ linear momentum conservations in $y$ and $z$ directions, respectively, while $X_{10}$ represents rotation in $y z$ plane.

\section{Discussion and Conclusions}

From our investigation we find that the Noether symmetries of the Lagrangian form a maximal set of $\mathbf{1 7}$ conservation laws. Since Milne metric represents a flat cosmological model, it admits maximal isometries. It is shown that the Noether symmetries for the Milne model give 7 additional conservation laws which are not given by the symmetries of the spacetime metric [13]. In exactly similar fashion, we find that the wave equation on the Milne metric also admits maximal group of isometries. It suggests that if the Lorentzian metric is flat, both Noether and Lie point symmetry groups will be maximal. It may be worth mentioning that the number of Noether symmetries in this study differs with the one given in [12]. It will be interesting to extend such investigations to more general Lorentzian metrics. It is hoped that such investigations will add to our understanding of Lie and Noether point symmetries for such metrics [13]. 


\section{Acknowledgment}

The authors would like to thank King Fahd University of Petroleum and Minerals for facilities to complete this work.

\section{References}

[1] E. Noether, "Invariante variationsprobleme, nachrichten der akademie der wissenschaften in Göttingen," Mathematisch-Physikalische Klasse, vol. 2, pp. 235-257, 1918, (English translation in Transport Theory and Statistical Physics, vol. 1, no. 3, pp. 186-207, 1971).

[2] R. Narain and A. H. Kara, "The Noether conservation laws of some Vaidiya metrics," International Journal of Theoretical Physics, vol. 49, no. 2, pp. 260-269, 2010.

[3] A. H. Kara and F. M. Mahomed, "Noether-type symmetries and conservation laws via partial Lagrangians," Nonlinear Dynamics, vol. 45, no. 3-4, pp. 367-383, 2006.

[4] A. H. Bokhari, A. H. Kara, A. R. Kashif, and F. D. Zaman, "Noether symmetries versus Killing vectors and isometries of spacetimes," International Journal of Theoretical Physics, vol. 45, no. 6, pp. 1029-1039, 2006.

[5] A. H. Bokhari and A. H. Kara, "Noether versus Killing symmetry of conformally flat Friedmann metric," General Relativity and Gravitation, vol. 39, no. 12, pp. 2053-2059, 2007.

[6] T. Feroze, "New conserved quantities for the spaces of different curvatures," Modern Physics Letters A, vol. 25, no. 13, pp. 1107-1114, 2010.

[7] A. D. Rendall, "Applications of the theory of evolution equations to general relativityin General Relativity," in Proceedings of the 16th International Conference, N. T. Bishop and S. D. Maharaj, Eds., World Scientific, Singapore, 2002.

[8] G. W. Bluman and S. C. Anco, Symmetry and Integration Methods for Differential Equations, Springer, New York, NY, USA, 2002.

[9] G. W. Bluman and S. Kumei, Symmetries and Differential Equations, vol. 81, Springer, New York, NY, USA, 1989.

[10] B. J. Cantwell, Introduction to Symmetry Analysis, Cambridge University Press, Cambridge, UK, 2002.

[11] P. E. Hydon, Symmetry Methods for Differential Equations, Cambridge University Press, Cambridge, UK, 2000.

[12] S. Jamal, A. H. Kara, and A. H. Bokhri, "Symmetries, conservation laws and reduction of wave and Gordon-type equations on Riemannian manifolds," World Academy of Science, Engineering and Technology, vol. 60, 2011.

[13] C. W. Misner, K. S. Thorne, and J. A. Wheeler, Gravitation, W. H. Freeman, San Francisco, Calif, USA, 1973. 


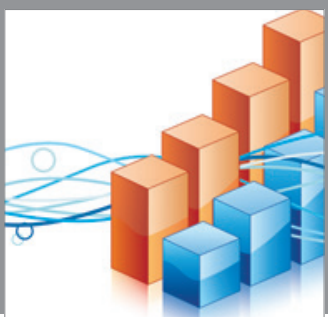

Advances in

Operations Research

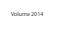

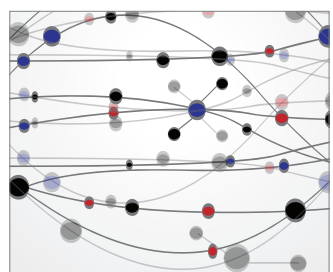

\section{The Scientific} World Journal
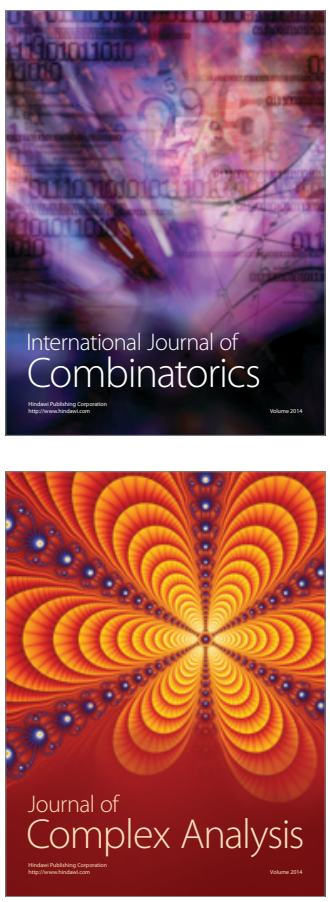

International Journal of

Mathematics and

Mathematical

Sciences
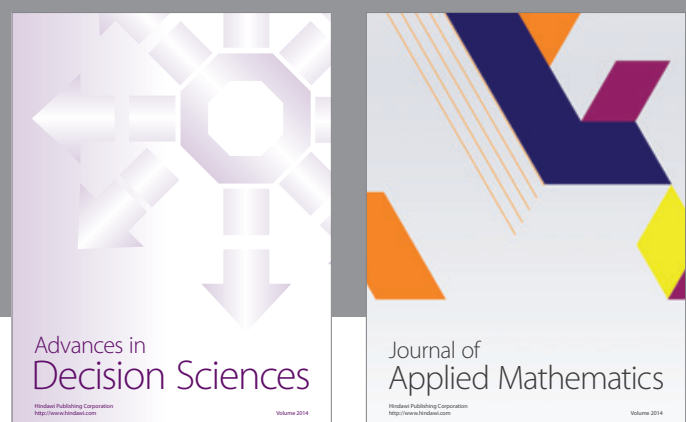

Journal of

Applied Mathematics
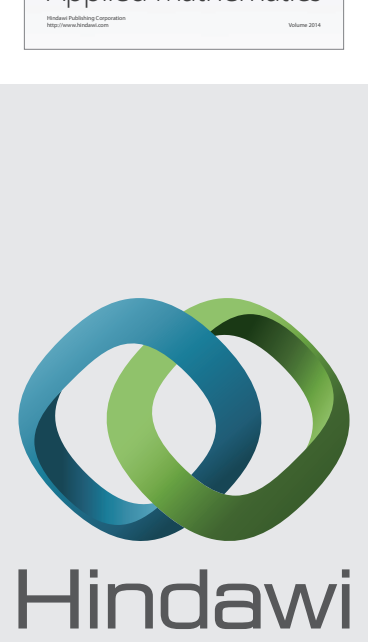

Submit your manuscripts at http://www.hindawi.com
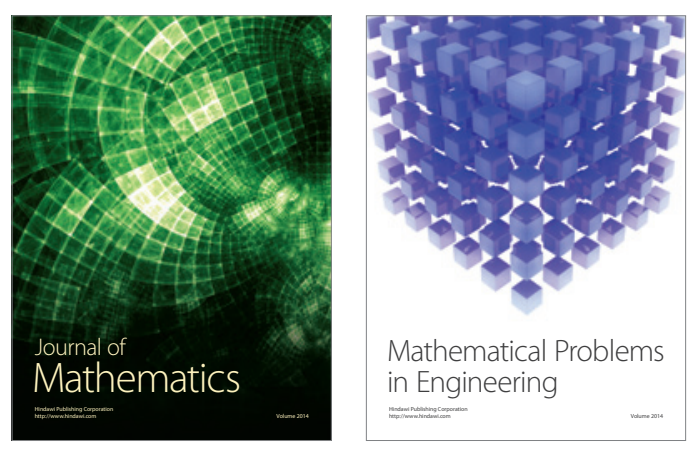

Mathematical Problems in Engineering
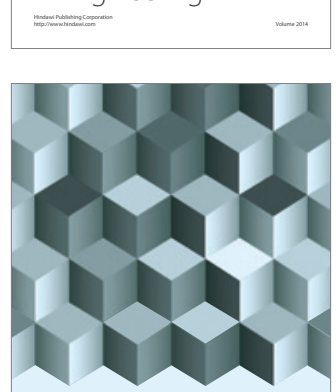

Journal of

Function Spaces
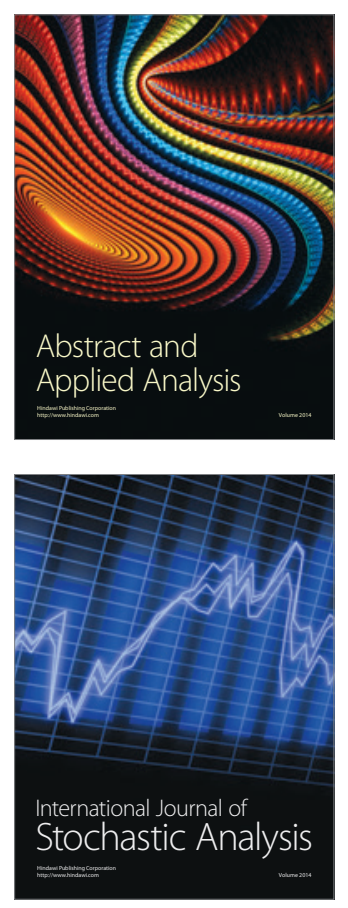

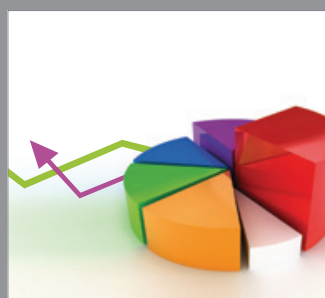

ournal of

Probability and Statistics

Promensencen
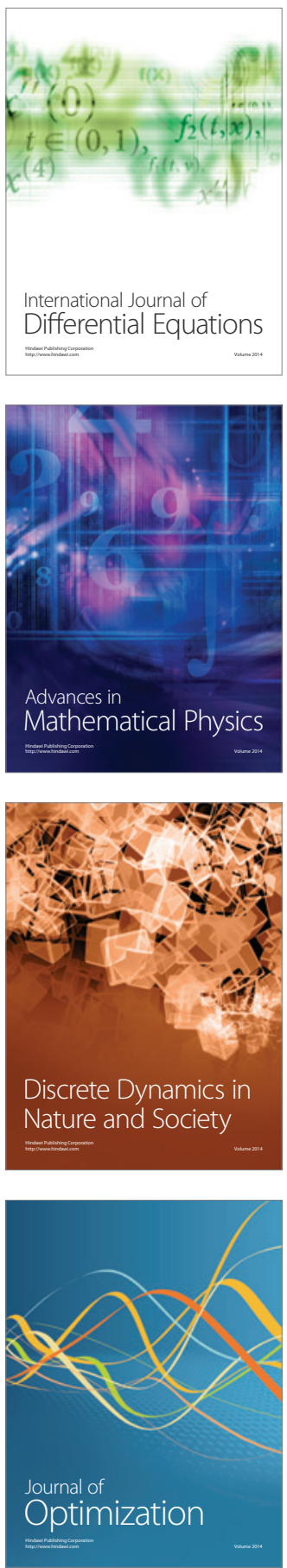